\title{
Harmonic Balance Analysis of the Generalized Chua's Circuit.
}

Article in International Journal of Bifurcation and Chaos · August 2006

DOI: $10.1142 /$ S0218127406016082 Source: DBLP

CITATIONS

13

2 authors:

\section{F.A. Savaci}

Izmir Institute of Technology

37 PUBLICATIONS 183 CITATIONS

SEE PROFILE
READS

50

Some of the authors of this publication are also working on these related projects:

stochastic bifurcation of generalized Chua's circuit, stochastic resonance in memristive systems, effect of alpha stable type stochastic loads on power lines, alpha stable noise as random carrier in communication systems View project 
International Journal of Bifurcation and Chaos, Vol. 16, No. 8 (2006) 2325-2332

(C) World Scientific Publishing Company

\title{
HARMONIC BALANCE ANALYSIS OF THE GENERALIZED CHUA'S CIRCUIT
}

\author{
F. ACAR SAVACI* \\ Izmir Institute of Technology, \\ Department of Electrical and Electronics Engineering, \\ Gülbahçe, Urla, Izmir, Turkey \\ acarsavaci@iyte.edu.tr \\ SERKAN GÜNEL \\ Dokuz Eylül University, \\ Department of Electrical and Electronics Engineering, \\ Tınaztepe Kampüsü, Kaynaklar, Buca, Izmir, Turkey \\ serkan.gunel@eee.deu.edu.tr
}

Received May 9, 2005; Revised August 11, 2005

\begin{abstract}
In this paper, the harmonic balance analysis of Generalized Chua's circuit exhibiting n-scroll attractors has been accomplished. The dual-input describing functions of the piecewise-linear characteristics of Chua's diode have been obtained and based on the harmonic balance principle, the existence and the locations of the n-scrolls have been verified.
\end{abstract}

Keywords: Generalized Chua's circuit; harmonic balance analysis; n-scroll attractors.

\section{Introduction}

Chua's circuit is a system for which chaos has been verified analytically [Chua et al., 1986; Silva, 1993]. Despite its minimal structure, it has a rich diversity of behaviors ranging from equilibrium states to period doubling bifurcations, from quasi-periodicity to intermittency which makes it a milestone in nonlinear research $\lceil$ Kennedy, 1993b, 1993c; Madan, 1993; Mira, 1997.

The importance of this circuit is that, every continuous three-dimensional odd-symmetric piecewiselinear vector field may be mapped onto this circuit, and hence, Chua's circuit can generate flows which are diffeomorphic to the flows of such vector fields [Chua et al., 1993]. The circuit has been actively studied and many realizations have been proposed (e.g. [Cruz \& Chua, 1992; Kennedy, 1993a; Kılıc et al., 2004; Matsumoto et al., 1985; Morgül,
1995; Senani \& Gupta, 1998; Zhong \& Ayron, 1985a]). The rich diversity of dynamical behaviors of the circuit have been experimentally verified in Matsumoto, 1984; Matsumoto et al., 1985; Zhong \& Ayron, 1985a, 1985b]. Furthermore, the circuit can be used as building blocks of Cellular Neural Networks [Chua \& Roska, 1993], and, static or dynamic coupling of these circuits may lead to a large variety of dynamic behavior [Dabrowski et al., 1993].

The generalization of Chua's circuit in the sense that the number and the position of the scrolls are altered without increasing the dimensionality of the system has been proposed by modification of the nonlinearity in Suykens, 1997; Suykens \& Vandewalle, 1991, 1993; Yalçın, 2004; Yalçın et al., 2001a, 2001b, 2000]. The comparable generalizations have been given in [Kataoka \& Saito, 1999; Kocarev et al., 1993; Madan, 1993; Saito, 1990; Wu \& Chua, 1996], however, in these papers either

\footnotetext{
*Author for correspondence.
} 
the models are hard to be realized or the dimension of the proposed models are higher than that of the original Chua's circuit.

It has been proposed in [Genesio \& Tesi, 1992] that the double scroll behavior of the circuit is due to the interacting limit cycles with unstable equilibrium point. The existence of interacting predicted limit cycles and unstable equilibrium points have been verified using heuristic harmonic balance method [Gelb \& Velde, 1967] in [Yalçn \& Savacı, 1999] for the basic form of the circuit with only two breakpoints.

In this paper, the harmonic balance analysis of the Generalized Chua's circuit exhibiting n-scroll attractors has been accomplished by extending the study in [Yalçın \& Savacı, 1999] and [Savacı et al., 2001]. In the first section of this study, the generalized Chua's circuit has been represented in the Lur'e form and the describing functions (DF) of the piecewiselinear characteristics with multiple break points of the nonlinear resistor have been obtained. In the subsequent sections, the existence and location of the n-scrolls have been verified based on the harmonic balance principle [Gelb \& Velde, 1967], and using the conjecture given in [Genesio \& Tesi, 1992].

\section{The Generalized Chua's Circuit}

Chua's circuit depicted in Fig. 1 has the following state representation

$$
\begin{aligned}
\dot{\mathbf{x}} & =\mathbf{A} \cdot \mathbf{x}+\mathbf{b} \cdot \phi(y) \\
y & =\mathbf{C} \cdot \mathbf{x}
\end{aligned}
$$

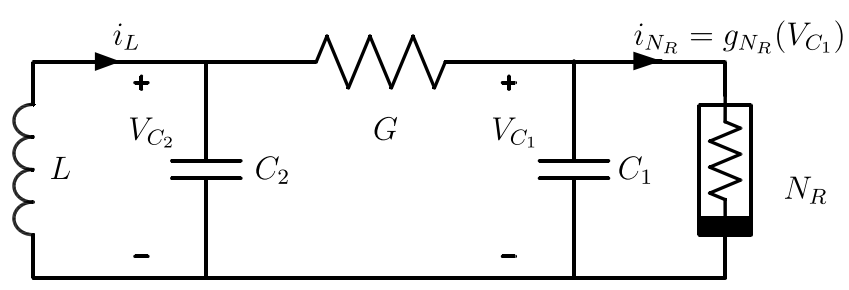

Fig. 1. Chua's circuit.

where

$$
\begin{gathered}
\phi(y)=m_{i} y+k_{i}, \quad S_{i}<y \leq S_{i+1} \\
k_{0}=0, \quad k_{i}=\sum_{j=1}^{i}\left(m_{j-1}-m_{j}\right) S_{j} \\
i= \pm 1, \pm 2, \ldots, \quad S_{0}=0 \\
\mathbf{A}=\left(\begin{array}{ccc}
-\alpha(1+\delta) & \alpha & 0 \\
1 & -1 & 1 \\
0 & -\beta & 0
\end{array}\right), \quad \mathbf{b}=\left(\begin{array}{c}
-\alpha \\
0 \\
0
\end{array}\right) \\
\text { and } \quad \mathbf{C}=\left(\begin{array}{lll}
1 & 0 & 0
\end{array}\right)
\end{gathered}
$$

where $\alpha, \beta$ and $\delta$ can be defined in terms of the circuit parameters.

The double scroll, i.e. the strange attractor of Chua's circuit, can be obtained for some specific range of parameters and with two breakpoints piecewise-linear resistor in the circuit (Fig. 2). In [Suykens et al., 1997], the n-scroll $(n=1,2, \ldots)$ attractor family was obtained as a result of generalization of Chua's circuit with additional breakpoints in the nonlinear characteristic of Chua's

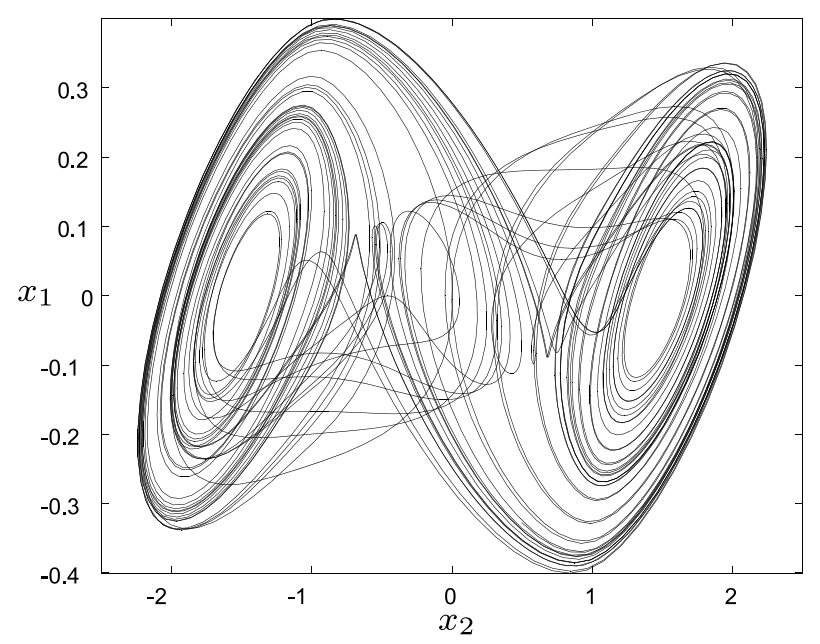

(b)

Fig. 2. The $\mathrm{v}-\mathrm{i}$ characteristic function of the nonlinear resistor in Chua's circuit and corresponding strange attractor, double scroll. $m_{1}=-(1 / 7), m_{2}=2 / 7, \alpha=9$ and $\beta=14.28$. 


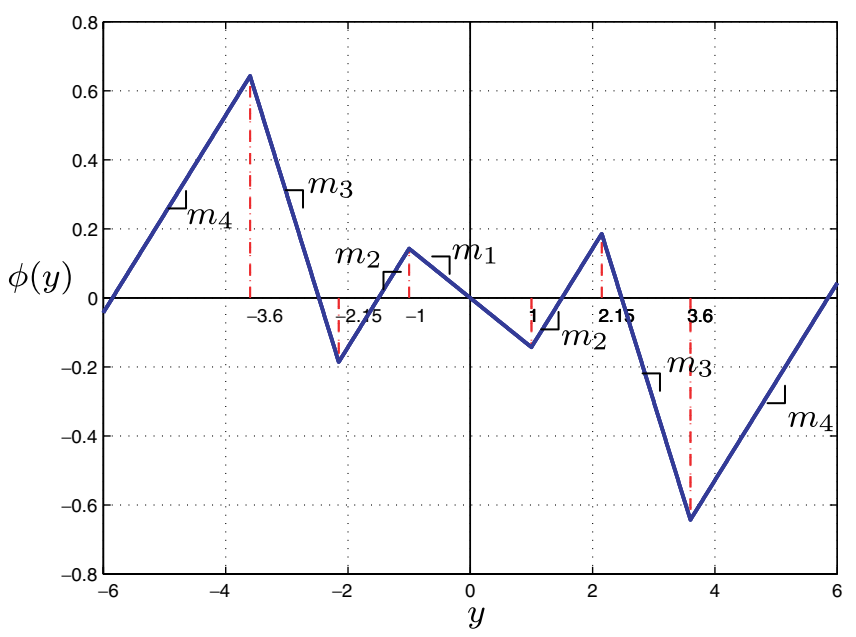

(a)

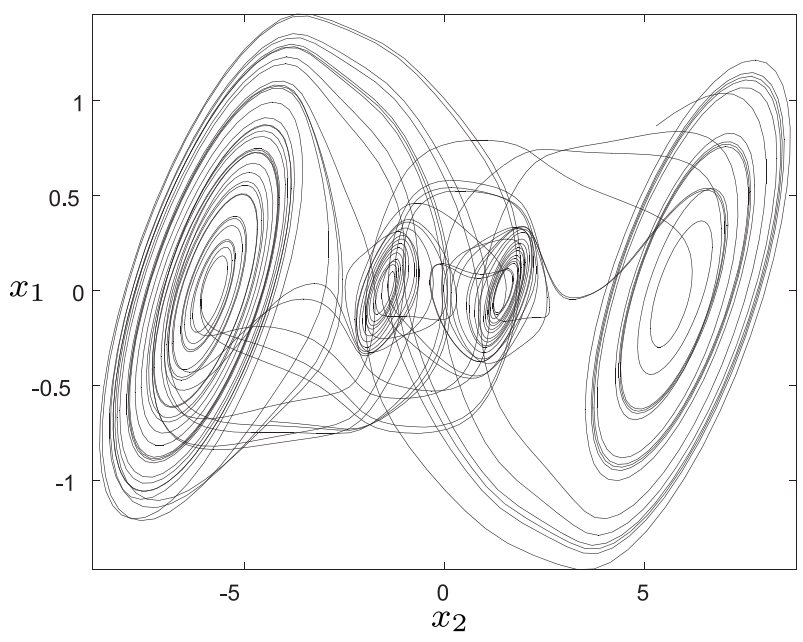

(b)

Fig. 3. The $\mathrm{v}-\mathrm{i}$ characteristic function of the diode with four breakpoints in the generalized Chua's circuit and corresponding strange attractor, 2-double scroll. $m_{1}=-(1 / 7), m_{2}=2 / 7, m_{3}=-(4 / 7), m_{4}=2 / 7, \alpha=9$ and $\beta=14.28$.

diode (Fig. 3). Due to the generalization of the nonlinear characteristics, it has been shown that increasing the number of scrolls in all state variable directions is also possible [Yalçın et al., 2001a].

\section{Harmonic Balance Analysis}

In order to analyze the circuit by harmonic balance method, the system is first represented in the classical Lur'e form (Fig. 4). The nonlinear part of the Lur'e form of Chua's circuit is as in (2) and the linear part can be represented as,

$$
L(s)=\frac{-\alpha\left(s^{2}+s+\beta\right)}{s^{3}+(1+\alpha(1+\delta)) s^{2}+(\beta+\alpha \delta) s+\alpha \beta(1+\delta)}
$$

Assume that the output of the system (1) is

$$
y(t)=A+B \sin (\omega t)
$$

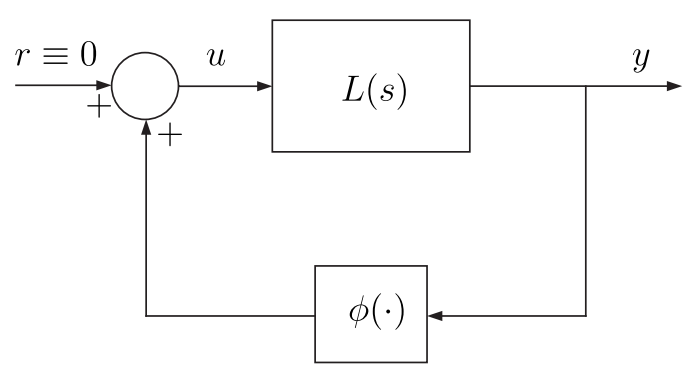

Fig. 4. The system in the Lur'e form. then the corresponding nonlinearity output is approximately given by

$$
\phi(y(t)) \approx N_{0}(A, B) A+N_{1}(A, B) B \sin (\omega t)
$$

where the approximating gain to the bias input is

$$
N_{0}(A, B)=\frac{1}{\pi} \int_{-\infty}^{\infty} \phi(A+B \sin (\omega t)) d t,
$$

and the approximating gain to the sinusoidal input is

$$
N_{1}(A, B)=\frac{1}{2 \pi} \int_{-\infty}^{\infty} \phi(A+B \sin (\omega t)) \sin (\omega t) d t .
$$

If $\phi: R \rightarrow R$ is memoryless, time invariant and odd with respect to its argument, then both dual input describing functions (DIDF), $N_{0}: R^{2} \rightarrow R$ and $N_{1}: R^{2} \rightarrow R$ are independent of $\omega$.

The approximation given in (6) is valid if

$$
|L(j \omega)| \gg|L(j k \omega)|, \quad k=2,3, \ldots, \quad j^{2}=-1
$$

hence the higher order harmonics generated by the nonlinearity can be neglected.

In order to have a limit cycle in the form of (5), the following equations must be satisfied

$$
\begin{aligned}
1+N_{0}(A, B) L(0) & =0 \\
1+N_{1}(A, B) L(j \omega) & =0
\end{aligned}
$$

To predict the limit cycles in all possible regions determined by the breakpoints, bias $A$, and the amplitude $B$ of the limit cycles have been 
considered. To locate all possible limit cycles the solutions of $\sum_{p=1}^{n}\left(\begin{array}{l}n \\ p\end{array}\right)$ nonlinear equations in the form

$$
\begin{array}{r}
\left.1+N_{0}^{(p, r)}(A, B) L(0)\right)=0 \\
1+N_{1}^{(p, r)}(A, B) L(j \omega)=0
\end{array}
$$

where $N_{0}^{(p, r)}, N_{1}^{(p, r)}$ are the gains of the nonlinearity which contains $p$ breakpoints $(p=1,2, \ldots, n)$ including $\left\{S_{r+i} \mid i=0,1, \ldots, p-1\right\}$ for $r=1$, $2, \ldots, n-p+1$, (Fig. 5).

For the nonlinearity including $p$ breakpoints in the region $S_{r-1}<y(t)<S_{r+p}, r=1,2, \ldots, n-$ $p+1$, the DIDFs have been obtained using a symbolic solver for the generalized Chua's diode as,

$$
\begin{aligned}
N_{0}^{(p, r)}(A, B)= & \frac{1}{2 A}\left[\left(m_{p+r}+m_{r}\right) A+k_{p+r}+k_{r}\right]+\frac{1}{A \pi} \sum_{i=1}^{p}\left\{\left[\left(m_{r+i}-m_{r+i-1}\right) A\right.\right. \\
+ & \left.\left.\left(k_{r+i}-k_{r+i-1}\right)\right] \sin ^{-1}\left(\frac{A-S_{r+i}}{B}\right)+\left(m_{r+i}-m_{r+i-1}\right) \sqrt{B^{2}-\left(A-S_{r+i}\right)^{2}}\right\} \\
N_{1}^{(p, r)}(A, B)= & \frac{1}{2}\left(m_{p+r}+m_{r}\right)+\frac{1}{\pi} \sum_{i=1}^{p}\left\{\left(m_{r+i}-m_{r+i-1}\right) \sin ^{-1}\left(\frac{A-S_{r+i}}{B}\right)\right. \\
& \left.+\frac{\left(m_{r+i}-m_{r+i-1}\right)\left(A+S_{r+i}\right)+2\left(k_{r+i}-k_{r+i-1}\right)}{B^{2}} \sqrt{B^{2}-\left(A-S_{r+i}\right)^{2}}\right\}
\end{aligned}
$$

To interpret the bifurcation phenomena, the stability of the located limit cycles has to be considered. Assuming that the bias is constant for small perturbations, the stability of the Predicted Limit

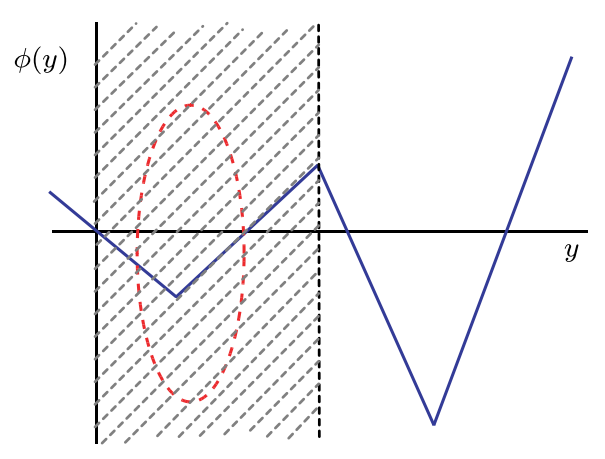

(a) $p=1, r=1$

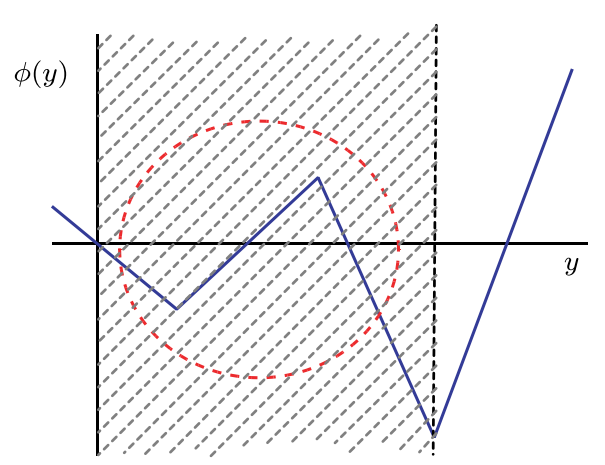

(c) $p=2, r=1$

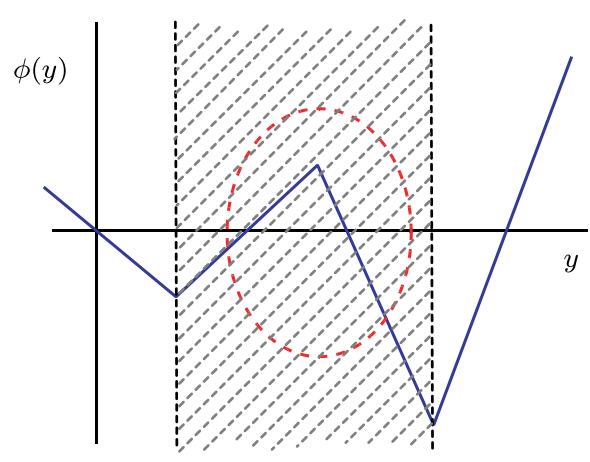

(b) $p=1, r=2$

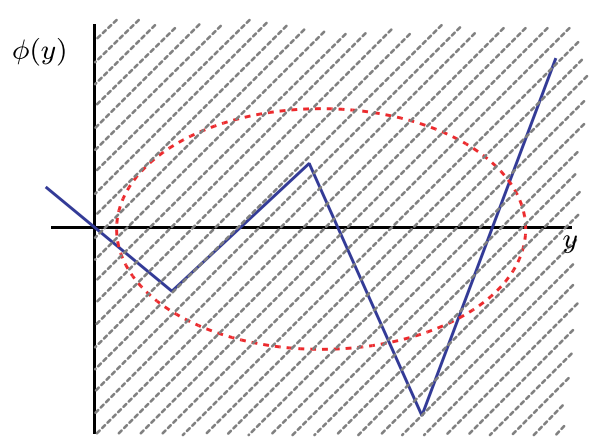

(d) $p=3, r=1$

Fig. 5. The various possible regions for predicted limit cycles. 
Cycles (PLC) can be studied via Loeb criteria [Gelb \& Velde, 1967; Ogata, 1990]. The relative location of the $-\left(1 / N_{1}\left(A_{0}, B\right)\right)$ locus with respect to the amplitude $B$ and the $L(j \omega)$ locus with respect to frequency $\omega$ provide the stability information of a limit cycle, and a sustained oscillation or a limit cycle occurs when $-\left(1 / N_{1}\left(A_{0}, B\right)\right)$ locus and $L(j \omega)$ locus intersect. If $-\left(1 / N_{1}\left(A_{0}, B\right)\right)$ is enclosed by the $L(j \omega)$ locus, then the system is unstable and the output amplitude subjected to any disturbance will increase, on the other hand, if it is not enclosed the system is stable, then the output amplitude will decrease (Fig. 6). Hence, by examining the locus in the range $\left|A-A_{0}\right| \leq \Delta A$ for the bias, the stability of predicted limit cycle can be concluded, where $A_{0}$ and $B_{0}$ are bias and amplitude of the PLC, respectively.

The instability of the PLC can also be verified analytically following the same line of reasoning in [Gelb \& Velde, 1967], which is stated for single sinusoidal input describing functions. Equation (10) can be represented as

$$
\begin{gathered}
U_{0}(A, B)=0 \\
U_{1}(A, B, \omega)+j V_{1}(A, B, \omega)=0
\end{gathered}
$$

where

$$
\begin{aligned}
U_{0}(A, B) & =1+N_{0}(A, B) L(0) \\
U_{1}(A, B, \omega) & =1+N_{1}(A, B) \mathfrak{R e}(L(j \omega)) \\
V_{1}(A, B, \omega) & =N_{1}(A, B) \mathfrak{I m}(L(j \omega))
\end{aligned}
$$

Consider a small simultaneous perturbation in amplitude, bias and the frequency as

$$
\begin{aligned}
& A \rightarrow A+\Delta A \\
& B \rightarrow B+\Delta B \\
& \omega \rightarrow \omega+\Delta \omega+j \Delta \sigma
\end{aligned}
$$

where $\Delta \sigma \triangleq-((d B / d t) / B)$. And the perturbed solution should still satisfy Eq. (10). Hence,

$$
\begin{aligned}
U_{0}(A+\Delta, B+\Delta B) & =0 \\
U_{1}(A+\Delta A, B+\Delta B, \omega+\Delta \omega+j \Delta \sigma) & =0 \\
V_{1}(A+\Delta A, B+\Delta B, \omega+\Delta \omega+j \Delta \sigma) & =0 .
\end{aligned}
$$

Taylor series expansion of Eq. (14), around the point $(A, B, \omega)$ rearranging and ignoring higher order terms yield to the first order,

$$
\begin{gathered}
\frac{\partial U_{0}}{\partial A} \Delta A+\frac{\partial U_{0}}{\partial B} \Delta B=0 \\
\frac{\partial U_{1}}{\partial \omega} \Delta \omega-\frac{\partial V_{1}}{\partial \omega} \Delta \sigma+\frac{\partial U_{1}}{\partial B} \Delta B+\frac{\partial U_{1}}{\partial A} \Delta A \\
+j\left(\frac{\partial V_{1}}{\partial \omega} \Delta \omega+\frac{\partial U_{1}}{\partial \omega} \Delta \sigma\right. \\
\left.+\frac{\partial V_{1}}{\partial B} \Delta B+\frac{\partial V_{1}}{\partial A} \Delta A\right)=0 .
\end{gathered}
$$

Eliminating $\Delta \omega$ in the real and imaginary parts of Eq. (17b) gives,

$$
\begin{aligned}
\left(\frac{\partial U_{1}}{\partial \omega}\right)^{2}+\left(\frac{\partial V_{1}}{\partial \omega}\right)^{2}= & -\frac{\Delta B}{\Delta \sigma}\left(\frac{\partial U_{1}}{\partial \omega} \frac{\partial V_{1}}{\partial B}-\frac{\partial V_{1}}{\partial \omega} \frac{\partial U_{1}}{\partial B}\right) \\
& -\frac{\Delta A}{\Delta \sigma}\left(\frac{\partial U_{1}}{\partial \omega} \frac{\partial V_{1}}{\partial A}-\frac{\partial V_{1}}{\partial \omega} \frac{\partial U_{1}}{\partial A}\right) .
\end{aligned}
$$
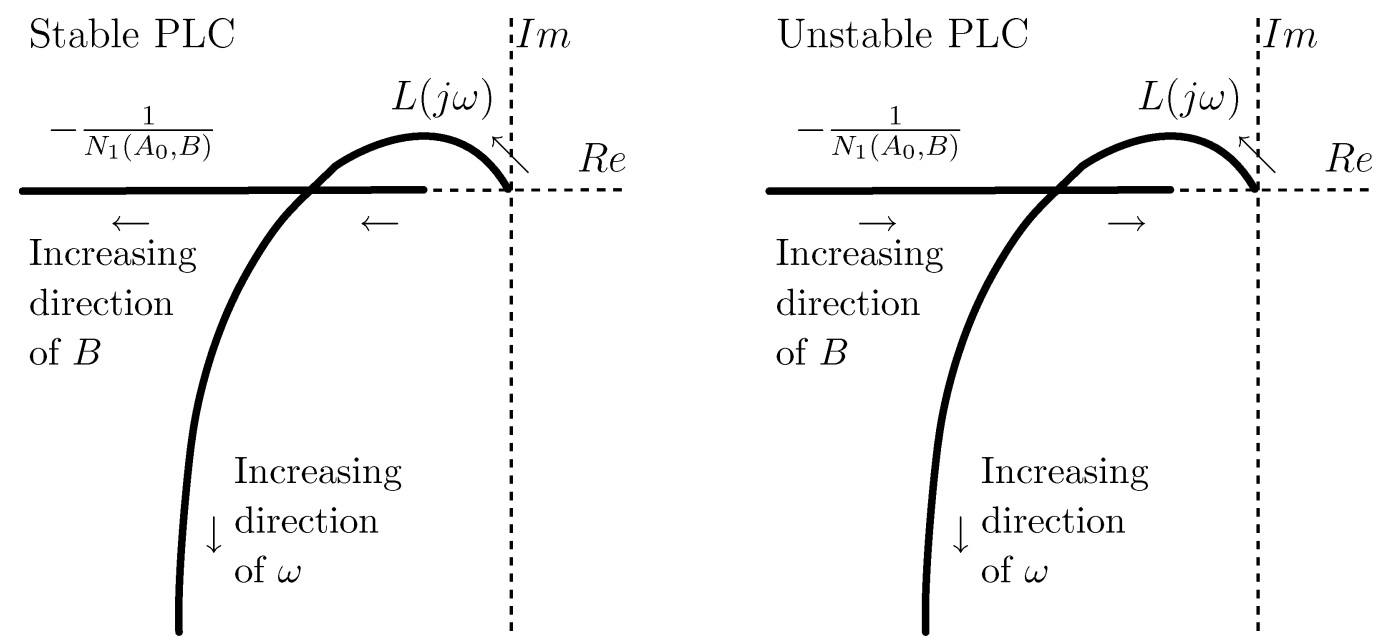

Fig. 6. The schematized interpretation of the Loeb criteria. 
$\Delta A$ in Eq. (18) can be eliminated using Eq. (17a) to give,

$$
\begin{aligned}
\left(\frac{\partial U_{1}}{\partial \omega}\right)^{2}+ & \left(\frac{\partial V_{1}}{\partial \omega}\right)^{2} \\
= & \frac{\Delta B}{\Delta \sigma}\left\{\frac{\frac{\partial U_{0}}{\partial B}}{\frac{\partial U_{0}}{\partial A}}\left(\frac{\partial U_{1}}{\partial \omega} \frac{\partial V_{1}}{\partial A}-\frac{\partial V_{1}}{\partial \omega} \frac{\partial U_{1}}{\partial A}\right)\right. \\
& \left.-\left(\frac{\partial U_{1}}{\partial \omega} \frac{\partial V_{1}}{\partial B}-\frac{\partial V_{1}}{\partial \omega} \frac{\partial U_{1}}{\partial B}\right)\right\}
\end{aligned}
$$

In order that the PLC is stable, the increment $\Delta B$ and $\Delta \sigma$ must have the same sign, i.e. $\Delta \sigma / \Delta B>0$. Since the left-hand side of Eq. (19) is always positive, it is necessary that,

$$
\begin{aligned}
& \frac{\frac{\partial U_{0}}{\partial B}}{\frac{\partial U_{0}}{\partial A}}\left(\frac{\partial U_{1}}{\partial \omega} \frac{\partial V_{1}}{\partial A}-\frac{\partial V_{1}}{\partial \omega} \frac{\partial U_{1}}{\partial A}\right) \\
& \quad-\left.\left(\frac{\partial U_{1}}{\partial \omega} \frac{\partial V_{1}}{\partial B}-\frac{\partial V_{1}}{\partial \omega} \frac{\partial U_{1}}{\partial B}\right)\right|_{\substack{A=A_{0} \\
B=B_{0} \\
\omega=\omega_{0}}}>0
\end{aligned}
$$

for the predicted limit cycle to be stable, where $A_{0}$, $B_{0}$ and $\omega_{0}$ are bias, amplitude and frequency of the PLC, respectively.

The PLC (5) is said to be interacting with an equilibrium point $y(t)=E$, if,

$$
B \geq \eta|A-E|
$$

with $\eta \approx 1$ [Genesio \& Tesi, 1992].
For the n-scroll case, one expects to find $n$ PLCs interacting with one or more unstable equilibrium points, losing their stabilities as the level of interaction increases (i.e. the distance between the equilibrium points and the PLCs decreases).

\section{Computational Results}

Since the nonlinearity is symmetric about the origin, examining the system for $y>0$ will be sufficient. As an example, for $\alpha=9, \beta=14.286$ and,

$$
\begin{aligned}
& \phi(y) \\
& = \begin{cases}-0.143 y, & 0<|y| \leq 1 \\
0.286 y-0.429 \operatorname{sign}(y), & 1<|y| \leq 2.15 \\
-0.571 y+1.414 \operatorname{sign}(y), & 2.15<|y| \leq 3.60 \\
0.286 y-1.671 \operatorname{sign}(y), & 3.60<|y|\end{cases}
\end{aligned}
$$

have been chosen.

For the range $\delta \in(-1.000,-0.857)$, the circuit either exhibits limit cycles, single, double or 2-double scroll strange attractors. The computations indicate that there are several solutions satisfying the system of equations (11) for various regions. However, only the solutions for $(p, r)=$ $(1,1)$ and $(p, r)=(3,1)$ are interacting with the unstable equilibrium point at the origin after a critical value of $\delta$. These limit cycles cause the double scroll and the 2-double scroll behavior. The results for various values of $\delta$, the solutions for these predicted limit cycles have been summarized in Tables 1 and 2.

The stability of predicted limit cycles has been first examined using inequality (16). If the inequality (16) is true, the Loeb criteria has been examined

Table 1. Bias $A_{0}$, amplitude $B_{0}$, frequency $\omega_{0}$ of the PLC and the stability of the PLC for the regions $(p, r)=(1,1)$.

\begin{tabular}{c|ccccc|c}
\hline$\delta$ & $\pm A_{0}$ & $B_{0}$ & $\omega_{0}$ & $\eta$ & $\begin{array}{c}\text { Stability } \\
\text { of the PLC }\end{array}$ & Simulation \\
\hline-0.885 & 0.84900 & 0.38142 & 2.45069 & 0.53242 & stable & period-1 cycle \\
-0.890 & 0.84058 & 0.42370 & 2.45069 & 0.58312 & stable & period-1 cycle \\
-0.920 & 0.83814 & 0.62051 & 2.45069 & 0.78237 & stable & period-1 cycle \\
-0.925 & 0.84321 & 0.64798 & 2.45069 & 0.80477 & unstable & period-2 cycle \\
-0.940 & 0.86505 & 0.72618 & 2.45069 & 0.86115 & unstable & period-2 cycle \\
-0.945 & 0.87433 & 0.75136 & 2.45069 & 0.87703 & unstable & period-4 cycle \\
-0.950 & 0.88454 & 0.77627 & 2.45069 & 0.89173 & unstable & period-n cycle \\
-0.975 & 0.94892 & 0.90011 & 2.45069 & 0.95119 & unstable & single-scroll \\
-0.980 & 0.96444 & 0.92527 & 2.45069 & 0.96083 & unstable & double-scroll \\
-0.995 & 1.01652 & 1.00274 & 2.45069 & 0.98622 & unstable & double-scroll \\
-1.000 & 1.03580 & 1.02943 & 2.45069 & 0.99363 & unstable & 2 double-scroll \\
\hline
\end{tabular}


Table 2. Bias $A_{0}$, amplitude $B_{0}$, frequency $\omega_{0}$ of the PLC and the stability of the PLC for the regions $(p, r)=(3,1)$.

\begin{tabular}{c|ccccc|c}
\hline$\delta$ & \multicolumn{7}{|c}{$A_{0}$} & $B_{0}$ & $\omega_{0}$ & $\eta$ & $\begin{array}{c}\text { Stability } \\
\text { of the PLC }\end{array}$ & Simulation \\
\hline-0.885 & 4.28648 & -3.35832 & 2.45069 & 0.07184 & unstable & period-1 cycle \\
-0.890 & 4.30688 & -3.39444 & 2.45069 & 0.08757 & unstable & period-1 cycle \\
-0.920 & 4.39377 & -3.64057 & 2.45069 & 0.24680 & unstable & period-1 cycle \\
-0.925 & 4.39763 & -3.68719 & 2.45069 & 0.28956 & unstable & period-2 cycle \\
-0.940 & 4.37371 & -3.84064 & 2.45069 & 0.46693 & unstable & period-2 cycle \\
-0.945 & 4.34887 & -3.89809 & 2.45069 & 0.54922 & unstable & period-4 cycle \\
-0.950 & 4.31201 & -3.96034 & 2.45069 & 0.64833 & unstable & period-n cycle \\
-0.955 & 4.25993 & -4.02926 & 2.45069 & 0.76933 & unstable & single scroll \\
-0.958 & 4.21967 & -4.07502 & 2.45069 & 0.85536 & unstable & single scroll \\
-0.960 & 4.18834 & -4.10792 & 2.45069 & 0.91958 & unstable & single scroll \\
-0.961 & 4.17119 & -4.12521 & 2.45069 & 0.95403 & unstable & single scroll \\
-0.962 & 4.15296 & -4.14314 & 2.45069 & 0.99019 & unstable & single scroll \\
\hline
\end{tabular}

to determine the stability for a range of bias values centered around $A_{0}$. For $\delta=-0.890$, both PLCs are far away from the origin and only $(p, r)=(1,1)$ is stable. Increasing $\delta$ until -0.920 the situation does not change. The limit cycles lose their stability after $\delta=-0.925$. In simulations, it has been observed that there exists a period-2 limit cycle at $\delta=-0.925$, and period-4 limit cycle, single scroll, double scroll and 2-double scroll with decreasing values of $\delta$.

The level of interaction can be determined using the distance between the projection of the predicted limit cycle on the $y$-axis and unstable equilibrium point at the origin, i.e. $d=\left|A_{0}-\right| B_{0}||$. When the bias $A_{0}$ and the amplitude $B_{0}$ values are examined, for decreasing values of $\delta$, an increasing interaction with the unstable equilibrium point origin is observed. The limit cycle indicated by $(p, r)=(3,1)$ is already in interaction with the origin around $\delta=-0.962$, and $(p, r)=(1,1)$ limit cycle interacts

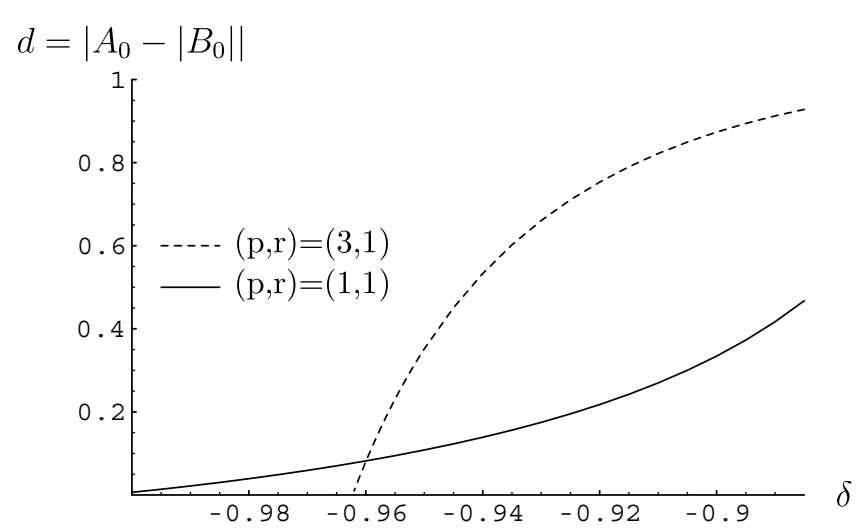

Fig. 7. The level of interaction as the bifurcation parameter $\delta$ is varied. with the origin around $\delta=1$ (Fig. 7). This confirms that the 2-double scroll behavior is due to the interaction of four limit cycles with unstable equilibrium point.

\section{Conclusions}

In this paper, it has been shown that it is possible to predict chaos in the generalized Chua's circuit exhibiting n-scroll attractors by harmonic balance analysis. To predict the interacting limit cycles, the dual input describing functions for n-breakpoint nonlinearity has been derived. The stability of predicted limit cycles have been examined graphically and also an necessary analytical condition for the stability of the predicted limit cycles has been given. The interaction of the unstable equilibria with nunstable predicted limit cycles is a strong indication of $n$-scroll chaos. The level of interaction has been measured using the distance between the interacting limit cycles and the equilibria. The usual period doubling route to chaos has been characterized by the increasing level of interaction.

\section{Acknowledgment}

Authors thank Mrs. Işıl İnkaya Yapalı for her kind support and valuable ideas to this study.

\section{References}

Chua, L. O., Komuro, M. \& Matsumoto, T. [1986] "The double scroll family," IEEE Trans. Circuits Syst. 33, 1072-1118.

Chua, L. O. \& Roska, T. [1993] "The CNN paradigm," IEEE Trans. Circuits Syst.-I: Fund. Th. Appl. 40, 147-156. 
Chua, L. O., Wu, C. W., Huang, A. \& Zhong, Z. Q. [1993] "A universal circuit for studying and generating chaos - Part I: Route to chaos and Part II: Strange attractors," IEEE Trans. Circuits Syst.-I: Fund. Th. Appl. 4, 745-761.

Cruz, J. M. \& Chua, L. [1992] "A CMOS IC nonlinear resistor for Chua's circuit," IEEE Trans. Circuits Syst.-I: Fund. Th. Appl. 39, 985-995.

Dabrowski, A. M., Dabrowski, W. R. \& Ogorzalek, M. J. [1993] "Dynamic phenomena in chain interconnections of Chua's circuit," IEEE Trans. Circuits Syst.-I: Fund. Th. Appl. 40, 868-871.

Gelb, A. \& Velde, W. E. V. [1967] Multiple-Input Describing Functions and Nonlinear System Design (McGraw Hill, NY).

Genesio, R. \& Tesi, A. [1992] "Harmonic balance methods for the analysis of chaotic dynamics in nonlinear systems," Automatica 28, 531-548.

Kataoka, M. \& Saito, T. [1999] "A 4-d chaotic oscillator with a hysteresis 2-port VCCS," in Proceedings of IEEE Int. Symp. Circuits and Systems (ISCAS2000), Vol. 5, pp. 418-421.

Kennedy, M. P. [1993a] "Synthesis of continuous piecewise-linear resistors for Chua's circuit family using operational amplifiers, diodes and linear resistors," Int. J. Circuit Th. Appl. 21, 551-558.

Kennedy, M. P. [1993b] "Three steps to chaos. I. Evolution," IEEE Trans. Circuits Syst.-I: Fund. Th. Appl. 40, 640-656.

Kennedy, M. P. [1993c] "Three steps to chaos. II. A Chua's circuit premier," IEEE Trans. Circuits Syst.-I: Fund. Th. Appl. 40, 657-674.

Kılıç, R., Cam, U., Alçı, M., Kuntman, H. \& Uzunhisarcikl, E. [2004] "Realization of inductorless Chua's circuit using FTFN-based nonlinear resistor and inductance simulator," Frequenz 58, 1-4.

Kocarev, L., Karadzinov, L. \& Chua, L. O. [1993] "ndimensional canonical Chua's circuit," J. Circuits Syst. Comput. 3, 239-258.

Madan, R. N. [1993] Chua's Circuit: A Paradigm for Chaos, World Scientific Series on Nonlinear Science, Series B, Vol. 3 (World Scientific, Singapore).

Matsumoto, T. [1984] "A chaotic attractor from Chua's circuit," IEEE Trans. Circuits Syst. 31, 1055-1058.

Matsumoto, T., Chua, L. \& Kumoro, M. [1985] "The double scroll," IEEE Trans. Circuits Syst. 32, 797818.

Mira, C. [1997] "Chua's circuit and the qualitative theory of dynamical systems," J. Franklin Inst. B 334, $737-744$.

Morgül, Ö. [1995] "Inductorless realization of Chua's circuit," Electron. Lett. 31, 1403-1404.

Ogata, K. [1990] Modern Control Engineering, 2nd edition (Prentice-Hall, NJ).
Saito, T. [1990] "An approach toward higher dimensional hysteresis chaos generators," IEEE Trans. Circuits Syst. 37, 399-409.

Savacı, F. A., Günel, S., Özkurt, N. \& Yapalı, I. İ. [2001] "Harmonic balance analysis of generalizad Chua's circuit," in Int. Conf. Progress in Nonlinear Science (Nizhny Novgorod, Russia).

Senani, R. \& Gupta, S. S. [1998] "Implementation of Chua's chaotic circuits using current feedback opmaps," Electron. Lett. 34, 829-830.

Silva, C. P. [1993] "The double hook attractor in Chua's circuit: Some analytical results," in Chua's Circuit: A Paradigm for Chaos, ed. Madan, R. N., Vol. 1 of World Scientific Series on Nonlinear Science, Series B, Vol. 1 (World Scientific, Singapore), pp. 671-710.

Suykens, J. A. K. \& Vandewalle, J. [1991] "Quasilinear approach to nonlinear systems and the design of ndouble scroll $(\mathrm{n}=1,2,3,4, \ldots)$," IEEE Proc. $G \mathbf{1 3 8}$, 595-603.

Suykens, J. A. K. \& Vandewalle, J. [1993] "Generation of N-double scrolls $(\mathrm{N}=1,2,3,4, \ldots)$," IEEE Trans. Circuits Syst.-I: Fund. Th. Appl. 40, 861-867.

Suykens, J. A. K., Huang, A. \& Chua, L. O. [1997] "A family of n-scroll attractors from a generalized Chua's circuit," AË̈ Int. J. Electron. Commun. 51, 131-138.

Wu, C. W. \& Chua, L. O. [1996] "On the generality of the unfolded Chua's circuit," Int. J. Bifurcation and Chaos 6, 801-832.

Yalçın, M. E. \& Savacı, F. A. [1999] "New realization of Chua's circuit and verification of chaos by harmonic balance analysis," ARI-An Int. J. Phys. Engin. Sci. 51, 169-174.

Yalçın, M. E., Suykens, J. A. K. \& Vandewalle, J. [2000] "Experimental confirmation of 3- and 5-scroll attractors from a generalized Chua's circuit," IEEE Trans. Circuits Syst.-I 47, 425-429.

Yalçın, M. E., Ozoğuz, S., Suykens, J. A. K. \& Vandewalle, J. [2001a] "2D grid scroll attractors," in Proc. NDES, The Netherlands, pp. 181-184.

Yalçın, M. E., Özoğz, S., Suykens, J. A. K. \& Vandewalle, J. [2001b] "Families of scroll grid attractors," Int. J. Bifurcation and Chaos 12, 23-41.

Yalçın, M. E. [2004] Cellular Neural Networks, MultiScroll Chaos and Syncronization: Theory, Applications and Implementations, $\mathrm{PhD}$ thesis, Katholieke Universiteit Leuven, Departement Elektrotechniek, Belguim.

Zhong, G. Q. \& Ayron, F. [1985a] "Experimental confirmation of chaos from Chua's circuit," Int. J. Circuit Th. Appl. 13, 93-98.

Zhong, G. Q. \& Ayron, F. [1985b] "Periodicity and chaos in Chua's circuit," IEEE Trans. Circuits Syst. 32, 501-503. 